\title{
Reducing the Incidence of Hospital-Associated Venous Thromboembolism Within a Network of Academic Hospitals: Findings From Five University of California Medical Centers
}

\author{
lan H. Jenkins, MD, SFHM', Richard H. White, MD², Alpesh N. Amin, MD, MBA, MACP, SFHM, FACC ${ }^{3}$, \\ Nasim Afsarmanesh, MD, SFHM ${ }^{4}$, Andrew D. Auerbach, MD, MPH ${ }^{5}$, Raman Khanna, MD ${ }^{5}$, Gregory A. Maynard, MD, MS, MHM ${ }^{6 *}$ \\ ${ }^{1}$ Department of Medicine, University of California San Diego Health System, San Diego, California; ${ }^{2}$ Department of Medicine, University of California \\ Davis, Davis, California; ${ }^{3}$ Department of Medicine, University of California, Irvine, Irvine, California; ${ }^{4}$ Department of Medicine, University of California \\ Los Angeles Health, Los Angeles, California; ${ }^{5}$ Department of Medicine, University of California, San Francisco, San Francisco, California; ${ }^{6}$ University \\ of California Davis Medical Center, Davis, California.
}

BACKGROUND: Almost 700 patients suffered from hospitalassociated venous thromboembolism (HA-VTE) across 5 University of California hospitals in calendar year 2011.

OBJECTIVE: Optimize venous thromboembolism (VTE) prophylaxis (VTEP) in adult medical/surgical inpatients and reduce HA-VTE by at least $20 \%$ within 3 years.

DESIGN: Prospective, unblinded, open-intervention study with historical controls.

SETTING: Five independent but cooperating academic hospitals.

PATIENTS: All adult medical and surgical inpatients with stays $\geq 3$ days. The baseline year was 2011,2012 to 2014 were intervention years, and year 2014 was the mature comparison period.

VTEP adequacy was assessed with structured chart review of 45 patients per month at each site via random selection beginning partway through the study. HA-VTE was identified by discharge coding, capturing patients readmitted within 30 days of prior VTE-free admit and VTE occurring during index admission. Cases were stratified medical versus surgical and cancer or noncancer.

INTERVENTIONS: Interventions included structured order sets with "3-bucket" risk-assessment, measure-vention, techniques to improve reliable administration of VTEP, and education.

RESULTS: Adequate prophylaxis reached $89 \%$ by early 2014. The rate of HA-VTE fell from $0.90 \%$ in 2011 to $0.69 \%$ in 2014 (24\% relative risk [RR] reduction; RR: $0.76,95 \%$ confidence interval: $0.68-0.852$ ), equivalent to averting 81 pulmonary emboli and 89 deep venous thrombi. VTE rates were highest in cancer and surgical patients.

CONCLUSIONS: Hospital systems can reduce HA-VTE by implementing a bundle of active interventions including structured VTEP orders with embedded risk assessment and measure-vention. Journal of Hospital Medicine 2016;11:S22-S28. (C) 2016 Society of Hospital Medicine
Venous thromboembolism (VTE), comprised of pulmonary embolism (PE) and deep vein thrombosis (DVT), impacts hundreds of thousands of Americans annually. ${ }^{1}$ The complications of VTE can be severe, including the post-thrombotic syndrome, pulmonary hypertension, and complications of anticoagulation. VTE is often a complication of hospitalization, and $\mathrm{PE}$ is a common preventable cause of hospital mortality. ${ }^{2,3}$ Pharmacologic VTE prophylaxis (VTEP) in atrisk patients is effective and endorsed by prominent guidelines. $^{4-6}$ However, VTEP is underutilized, with only $30 \%$ to $50 \%$ of eligible patients receiving the right drug, dose, and duration. ${ }^{7,8}$

\footnotetext{
*Address for correspondence and reprint requests: Gregory A. Maynard, MD, Clinical Professor of Medicine and CQO, University of California Davis Medical Center, 2315 Stockton Blvd., Sherman Building Suite 2600, Sacramento, CA 95817; Telephone: 916-434-1166; Fax: 916-734-3234; E-mail: gmaynard@ucdavis.edu

Additional Supporting Information may be found in the online version of this article.

Received: June 15, 2016; Revised: September 2, 2016; Accepted: September 11, 2016

2016 Society of Hospital Medicine DOI 10.1002/jhm.2658

Published online in Wiley Online Library (Wileyonlinelibrary.com).
}

Public reporting and reimbursement policies reflect the magnitude of VTE as a public health concern. The Centers for Medicare and Medicaid Services (CMS) withholds incremental payment for VTE complications. ${ }^{9}$ The rate of hospital-associated VTE (HA-VTE) is used by benchmarking organizations as a quality indicator. ${ }^{10,11}$

The University of California (UC) has 5 major academic medical centers, located in Irvine (UCI), Los Angeles (UCLA), Sacramento (UC Davis [UCD]), San Diego (UCSD), and San Francisco (UCSF). In both 2010 and 2011, almost 700 UC patients suffered from HA-VTE annually. Barriers to optimal VTEP included the absence of standardized VTE risk assessment, lack of consensus on appropriate VTEP options for various inpatient populations, and a lack of collaborative infrastructure. Other barriers included poor adherence to mechanical prophylaxis and suboptimal measurement of prophylaxis and HA-VTE outcomes.

In late 2011, leaders from the 5 medical centers, supported by an internal competitive grant from the UC Office of the President and the Center for Health Quality and Innovation, formed a collaborative to 
address barriers, optimize VTEP in inpatients, and reduce HA-VTE across the system. Prior efforts at UCSD illustrated single-center improvement, with an increase in adequate VTEP from $50 \%$ to over $95 \%$, and a nearly $40 \%$ reduction in the incidence of HAVTE. ${ }^{12}$ We set out to scale this success across all 5 sites as a coordinated collaborative.

\section{METHODS}

This was a prospective, unblinded, open-intervention study with historical controls that assessed prespecified outcomes before, during, and after institution of multiple VTEP strategies in 5 independent, but cooperating, academic hospitals. All adult medical and surgical inpatients were included; psychiatric, obstetricsgynecology, rehabilitation, observation status, and pediatric populations were excluded. The study period was July 1, 2012 through June 30, 2015. Calendar year (CY) 2011 was the baseline year for comparison; interventions were initiated in CY 2012 to CY 2014, and CY 2014 was considered the mature postintervention period.

\section{Hospital Collaboration}

Multiprofessional teams ${ }^{1}$ were formed at each site. Monthly webinars, regular e-mail, minutes, and a project management plan with task lists were utilized for coordinated collaboration. Software (Dropbox) was used for sharing tools, educational materials, and measurement techniques. REDCap (Research Electronic Data Capture) was used for secure data collection and analysis of outcomes. ${ }^{13}$ Prior experience at UCSD and the Society of Hospital Medicine informed measurement and intervention bundle strategies. ${ }^{1,12,14}$ Surveys of baseline VTE prevention protocols, measures, and order sets were performed at each site. Measures were standardized, whereas the intervention bundle was tailored for use at each medical center. Institutional review board approval with a waiver for individualized informed consent was obtained.

\section{Interventions}

All sites were tasked with implementing a defined bundle of mutually reinforcing interventions that constituted a comprehensive VTE prevention program. These protocols, order sets, educational programs, and interventions were not designed or implemented in an identical fashion at each hospital, but common principles were utilized.

\section{VTE Prevention Protocol}

This protocol incorporated (1) standardized VTE risk assessment, and (2) links to a menu of appropriate prophylaxis options for each level of risk that included guidance for management of patients with contraindications to pharmacologic prophylaxis. We used simple risk-assessment models that grouped patients into 3 levels of risk (the 3-bucket model) rather than more complicated point-based systems. The 3-bucket model was designed to offer detailed guidance and avoid over-prophylaxis. Protocol, measurement, and order set tools were modified for special populations, such as orthopedic and neurosurgery populations. Operational definitions for bleeding risk, DVT risk, and exceptions to the protocol were explicit, which allowed for classification of adequate versus inadequate prophylaxis. High-risk patients required combination prophylaxis, moderate risk anticoagulant prophylaxis, and low risk patients no prophylaxis beyond ambulation protocols (in the absence of contraindications). Acceptable contraindications to pharmacologic prophylaxis included an international normalized ratio $>1.8$, platelet count $<50,000$, active hemorrhage within the last 3 days, known bleeding disorders, hypertensive urgencies/emergencies, comfort care-only status, and leeway times around surgery or other events $(24$ hours for most surgeries, 48 hours for transplant surgery or major trauma, up to a week after central nervous system surgery). Impaired mobility was considered present unless the patient could ambulate independently more than once a day. More details regarding 3-bucket risk models and explicit criteria can be reviewed in a recent Agency for Healthcare Quality and Research (AHRQ) publication. ${ }^{1}$ The protocol was embedded into clinical decision-support as required elements of admission, transfer, and postoperative order sets.

\section{Educational Programs}

Nurse and physician education programs were developed that stressed the importance of VTE prevention and adherence to thromboprophylaxis, including mechanical prophylaxis. The VTEP protocol was socialized in medical staff and nursing meetings. The educational programs recommended imaging only the proximal veins in patients with symptoms of leg DVT, and avoiding screening ultrasounds in asymptomatic patients. Physicians were coached on how to use the VTEP order sets. Content for educational programs was discussed and often shared among sites, but educational programs were tailored locally to fit perceived needs and available resources.

\section{Measure-vention}

An active surveillance and feedback program called measure-vention was developed to provide ongoing feedback to care providers regarding the appropriate use of VTEP over the duration of hospitalization. Key features of measure-vention were regular measurement of adherence/lapses in VTEP delivery, coupled with concurrent intervention to correct any lapses, with a nurse/pharmacist calling the primary team if VTEP was suboptimal. ${ }^{1,12}$ Measure-vention was utilized to monitor both appropriateness of orders and adherence with ordered prophylaxis, and was used to correct overprophylaxis as well as underprophylaxis. For 
example, our protocol specified that moderate VTE risk patients with a captured contraindication to anticoagulant should be on mechanical prophylaxis. An intervention would take place if mechanical prophylaxis was not ordered, or if it was ordered but not documented as being in place. Measure-vention examples and further description are available in AHRQ publications. $^{1}$

\section{Outcomes}

\section{Thromboprophylaxis Rates}

We planned to perform structured chart review on at least 30 non-critical care and 15 critical care adult inpatients per month at each site. Adult inpatients with a length of stay $>48$ hours, stratified by critical care versus non-critical care status, were assigned a numeric value by a random number generator. Patients were selected in order of random number assignment for chart review until the desired number of audits was completed. Development of the audit tools, as well as availability of personnel, led to delays in assessing prophylaxis rates by these standards until late 2012 to early 2013 at each site. A few sites had brief lapses in data collection during personnel changes. VTE risk, bleeding risk, prophylaxis ordered at the time of the audit, and adequacy of VTEP defined by a common standard were all assessed and recorded in the REDCap data repository. VTEP was considered adequate if combined pharmacologic and mechanical prophylaxis was present in the highest-risk patients or anticoagulant prophylaxis was present in moderate patients. Prophylaxis was considered adequate for all low-risk patients. Patients at risk for VTE with contraindications to anticoagulants were considered to be on adequate prophylaxis if they received mechanical prophylaxis or had documented contraindications to mechanical prophylaxis. The proper administration of ordered prophylaxis was scrutinized locally and targeted by education and other interventions at each site, but these data were not collated and analyzed centrally.

\section{Identification of HA-VTE}

HA-VTE rates were determined by administrative coding data, using International Classification of Diseases, Ninth Revision, Clinical Modification (ICD9-CM) codes in a manner similar to AHRQ Patient Safety Indicator 12 identification of postoperative VTE cases. ${ }^{10}$ Data were submitted by each hospital, then collated and analyzed using data from Vizient (formerly the University HealthSystem Consortium). The incidence of VTE was determined using specific ICD-9-CM hospital discharge codes: for PE: 415.11, 415.13, 415.19, 673.24; and for DVT: proximal DVT: 451.11, 451.19, 451.81, 453.41; distal DVT: 453.42; and other DVT: 453.40, 453.8. These codes have high positive predictive value for acute VTE. ${ }^{15,16}$ Mean age, average length of stay (ALOS), and admission severity of illness (SOI) scores were also captured from Vizient and summarized for the inpatient cohort each year.

All VTE cases were coupled with present on admission (POA) indicators. HA-VTE cases included patients who were readmitted to the same hospital within 30 days for a new event $(\mathrm{POA}=\mathrm{Y}$, but readmitted), as well as patients who developed PE or DVT during their hospitalization $(\mathrm{POA}=\mathrm{N}$ or $\mathrm{U})$. Only patients hospitalized for 3 or more days were analyzed for inpatient development of VTE, as diagnosis of VTE in the first 2 days was deemed either likely present on admission or not preventable using VTEP started within 24 hours of admission. VTE outcomes were assigned in a hierarchical fashion: if both PE and DVT were present, the case was classified as PE. Distal DVT was distinguished from proximal DVT whenever possible. Cases were stratified based on whether the patient had undergone a major operation (surgery patients) or not (medical patients). This stratification was based on the Medicare Severity- DiagnosisRelated Group (MS-DRG) coded in patient records. The DRG type for each MS-DRG was based on the 2015 CMS-MS-DRG codes for major operations, ${ }^{9}$ except that all trauma cases were considered surgical, and cases with vena cava filter placement and no other surgical procedure were considered medical. Cancer cases were identified using ICD-9-CM codes 140.00209.99 and 210.00-239.99.

\section{Review of HA-VTE}

Periodic review of selected HA-VTE cases identified by administrative coding data was recommended as a best practice, potentially adding insight to contributing factors to HA-VTE, included lapses in prophylaxis and suboptimal mobilization. The accuracy of diagnostic coding, and assessment of how HA-VTE cases were identified (symptoms vs screening ultrasounds) could also be assessed. Examples of audit tools were shared. Every site reviewed some HA-VTE cases, but the extent and duration of case review was left to the discretion of each site.

\section{Statistical Analysis}

Relative risk (RR) calculations with $95 \%$ confidence intervals $(\mathrm{CI})$ were used to compare the proportions of patients with PE, DVT alone, and total HA-VTE in 2014 versus 2011. The absolute risk reduction was multiplied by the population at risk in CY 2014 to arrive at estimates of cases of VTE averted in 2014 compared to 2011.

\section{RESULTS}

Robust sampling (421 to 728 patients at each site) revealed attainment of high rates of adequate VTE prophylaxis $(82 \%$ to $96 \%$ at all sites, collectively $89 \%$ ) by early 2014 . Common measures for adequate VTEP were not finalized and collected by all sites 
TABLE 1. Pulmonary and Deep Vein Thrombosis Rates Calendar Years 2011 to 2014

\begin{tabular}{|c|c|c|c|c|c|c|}
\hline & $\begin{array}{c}2011 \text { (Baseline), } \\
\text { No./\% }\end{array}$ & $\begin{array}{l}2012, \\
\text { No./\% }\end{array}$ & $\begin{array}{l}2013, \\
\text { No./\% }\end{array}$ & $\begin{array}{l}2014 \text { (Mature), } \\
\quad \text { No./\% }\end{array}$ & $\begin{array}{c}2014 \text { Versus } 2011 \\
\text { Relative Risk }(95 \% \text { Cl) }\end{array}$ & $\begin{array}{c}2014 \text { Versus } 2011 \\
\text { Estimated Averted } \\
\text { Events (95\% Cl) }\end{array}$ \\
\hline Total discharges (medical and surgical) & 73,941 & 76,100 & 77,300 & 79,565 & & \\
\hline Total PE + leg DVT & $667 / 0.90 \%$ & $650 / 0.85 \%$ & $568 / 0.73 \%$ & $546 / 0.69 \%$ & $0.761(0.680-0.852)$ & $170(103-247)$ \\
\hline Total PE & $363 / 0.49 \%$ & $359 / 0.47 \%$ & $340 / 0.44 \%$ & $309 / 0.39 \%$ & $0.791(0.680-0.920)$ & $81(32-135)$ \\
\hline Total leg DVT & $304 / 0.41 \%$ & $291 / 0.38 \%$ & $228 / 0.29 \%$ & $237 / 0.3 \%$ & $0.725(0.612-0.858)$ & $89(40-135)$ \\
\hline Medical discharges & 31,219 & 32,597 & 33,805 & 34,875 & & \\
\hline Total PE + leg DVT & $178 / 0.57 \%$ & $168 / 0.52 \%$ & $164 / 0.49 \%$ & $179 / 0.51 \%$ & $0.900(0.732-1.1071)$ & \\
\hline PE & $110 / 0.35 \%$ & $94 / 0.29 \%$ & $106 / 0.31 \%$ & $104 / 0.30 \%$ & $0.846(0.648-1.106)$ & \\
\hline Leg DVT & $68 / 0.22 \%$ & $74 / 0.23 \%$ & $58 / 0.17 \%$ & $75 / 0.22 \%$ & $0.987(0.711-1.371)$ & \\
\hline Surgical discharges & 42,722 & 43,503 & 43,495 & 44,690 & & \\
\hline Total PE + leg DVT & $489 / 1.14 \%$ & $482 / 1.11 \%$ & $404 / 0.93 \%$ & $367 / 0.82 \%$ & $0.718(0.627-0.821)$ & \\
\hline PE & $253 / 0.59 \%$ & $265 / 0.61 \%$ & $234 / 0.54 \%$ & $205 / 0.46 \%$ & $0.775(0.645-0.931)$ & \\
\hline Leg DVT & $236 / 0.55 \%$ & $217 / 0.50 \%$ & $180 / 0.41 \%$ & $162 / 0.36 \%$ & $0.656(0.538-0.801)$ & \\
\hline
\end{tabular}

NOTE: All adult Inpatients (with and without cancer diagnosis), discharged from all University of California Hospitals are included. The impact of improvement efforts was highest in the surgical population. See text for additional detail on proximal versus distal DVT. Abbreviations: $\mathrm{Cl}$, confidence interval; DVT, deep vein thrombosis; $\mathrm{PE}$, pulmonary embolism.

until early 2013, so we did not capture baseline VTEP rates, and could not compare baseline to mature prophylaxis rates. Reliable administration of mechanical and anticoagulant prophylaxis was monitored and targeted by each institution, albeit not in an identical fashion at each site. Adherence to mechanical prophylaxis was reported as improved at the sites, but these data were not collated and analyzed centrally.

\section{Population Demographics and Severity of Illness}

There were 73,941 to 79,565 discharges that met the criteria (adult medical-surgical inpatient with $>2$ day length of stay each year. Mean age and ALOS were unchanged or had no change of clinical significance. For example, in 2011 versus 2014, mean age was 55.7 versus 56.4 years, and ALOS was identical in both time periods at 7.4 days. Admission SOI scores also remained fairly static from 2011 to 2014 (2.27, $2.31,2.32,2.26$, respectively), and the admission SOI was not statistically different in 2011 versus 2014 (estimated difference of 2 means $0.01,95 \%$ CI: 0.00 0.02).

\section{Hospital-Associated VTE}

There were 2431 HA-VTE events observed in 306,906 adult inpatients across CY 2011 to 2014 (Table 1). The baseline incidence of HA-VTE was $0.90 \% \quad(667$ events in 73,941 hospitalizations in 2011). The incidence of HA-VTE in the postintervention period was $0.69 \%$ (546 HA-VTE events in 79,565 hospitalizations in $2014, P<0.001$ ), an overall reduction of $24 \%$. The absolute risk for PE decreased from $0.49 \%$ to $0.39 \%$ (RR: $0.79,95 \%$ CI: $0.68-0.92$ ), a reduction of $21 \%$, and the absolute risk of leg DVT fell from $0.41 \%$ to $0.30 \%$ (RR: 0.73 , 95\% CI: 0.61-0.86), a reduction of $27 \%$. Both proximal and distal DVT were reduced significantly. Proximal DVT was much more commonly diagnosed than distal DVT. Proximal DVT incidence decreased from
$0.32 \%$ to $0.25 \%$ (RR: $0.77,95 \%$ CI: $0.64-0.93$ ), whereas distal DVT incidence decreased from $0.09 \%$ to $0.05 \%$ (RR: $0.58,95 \%$ CI: $0.39-0.86$ ). The lower overall VTE rate in the postimplementation period compared with the baseline period corresponds to an estimated 170 fewer cases of VTE per year (89 DVT, $81 \mathrm{PE})$.

The baseline rate of HA-VTE and degree of improvement varied between institutions (Figure 1). UCI and UCD began the study with significantly higher VTE rates, and enjoyed the largest improvements. UCLA's VTE rate decreased to a lesser extent, whereas UCSD and UCSF rates remained relatively flat or were marginally higher. In contrast to the highly variable 2011 baseline rate of HA-VTE $(0.60 \%-$ $1.36 \%$ ), all 5 sites had HA-VTE rates within a very narrow range $(0.65 \%-0.73 \%)$ at maturity in 2014 .

\section{Cancer Versus Noncancer Patients}

The incidence of HA-VTE was higher in cancer patients than in noncancer patients. In 2011, 227 of $18,487(1.23 \%)$ cancer patients developed VTE, versus 440 of $55,454(0.79 \%)$ noncancer patients (Figure 2). After implementation of the VTE prevention initiative, the incidence of VTE in cancer patients fell by $0.21 \%(210$ events in 20,544 patients in 2014, $1.02 \%)$, and the incidence of VTE in noncancer patients fell by $0.22 \%$ (336 events in 59,021 patients, $0.57 \%)$. The relative risk of HA-VTE after the VTE interventions was reduced by $17 \%$ (RR: $0.83,95 \%$ CI: $0.69-1.00$ ) in cancer patients and 28\% (RR: 0.72 , 95\% CI: 0.62-0.83) in noncancer patients.

\section{Surgical Versus Medical Patients}

The impact of the VTE prevention initiative was only significant in surgical patients, for whom the risk of HA-VTE fell by $28 \%$ (RR: $0.72,95 \%$ CI: $0.63-0.82$ ) (Table 1). Medical patients experienced a nonsignificant $10 \%$ reduction in HA-VTE (RR: $0.90,95 \%$ CI: 


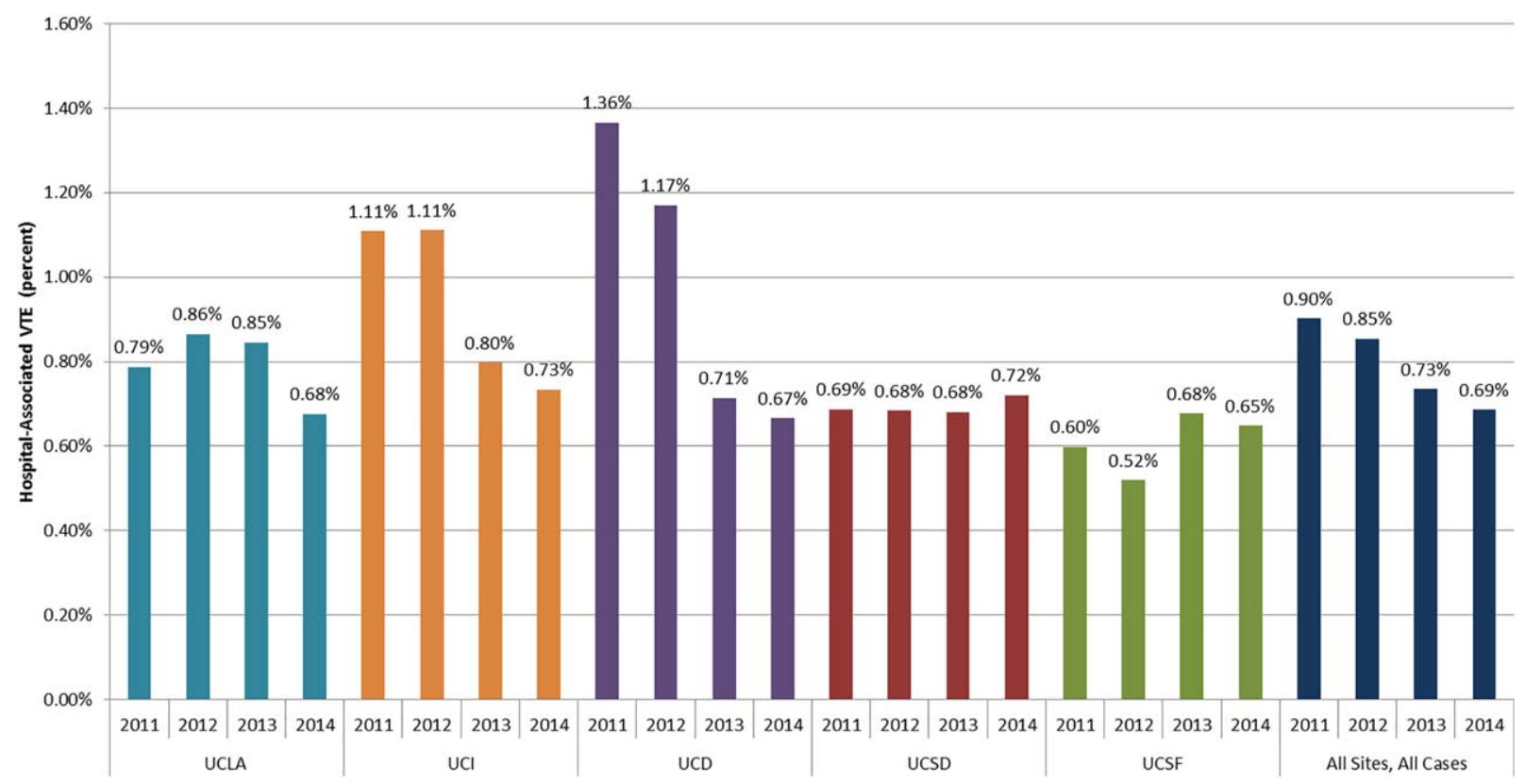

FIG. 1. Percent of adult inpatient discharges (with and without cancer) with a hospital-associated VTE $(N=306,906$ over 4 years $[132,495$ medical and 174,410 surgical patients]). Results for each site and as a composite are shown. Abbreviations: UCD, University of California, Davis; UCI, University of California, Irvine; UCLA, University of California, Los Angeles; UCSD, University of California, San Diego; UCSF, University of California, San Francisco; VTE, venous thromboembolism.

0.73-1.11). Medical patients had a significantly lower baseline incidence of HA-VTE $(0.57 \%)$ compared with surgical patients $(1.14 \%$; relative difference: $50 \%, P<0.001)$. This finding persisted postimplementation, with a cumulative incidence in medical patients of $0.51 \%$ versus $0.82 \%$ in surgical patients (relative difference: $31 \%, P<0.001$ ).

\section{DISCUSSION}

Our initiative, comprised of a collaborative infrastructure, a proven quality-improvement framework, and a

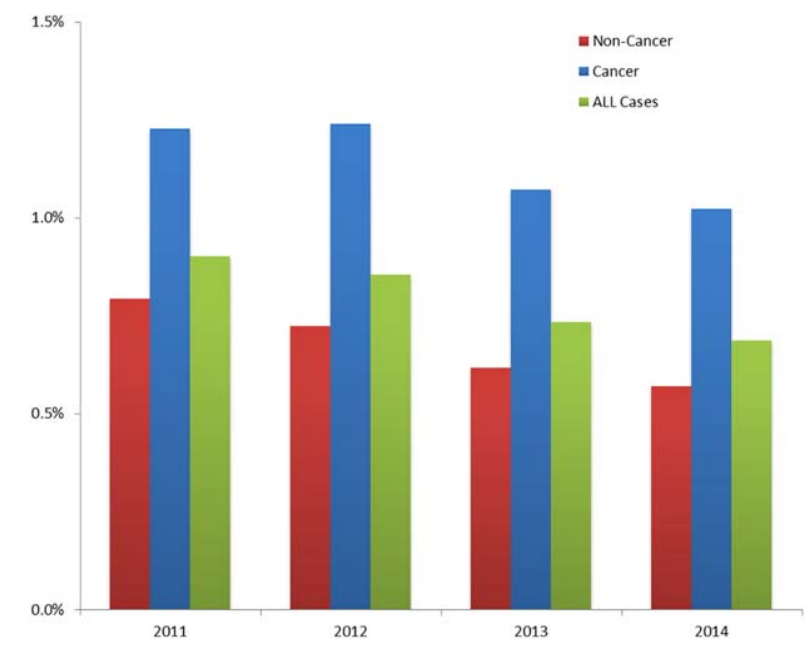

FIG. 2. Percent of adult inpatient discharges with HA VTE at all 5 University of California medical centers from 2011 to 2014. Patients with cancer ( $\mathrm{n}=$ 78,280 ) had higher rates of HA VTE than their counterparts without a diagnosis of cancer $(n=228,626)$. The risk of HA VTE was significantly reduced in calendar year 2014 versus the baseline calendar year 2011 (relative risk: 0.761 , 95\% confidence interval: 0.680-0.852). Abbreviations: HA VTE = hospital-associated venous thromboembolism. bundle of interventions, was associated with a $24 \%$ reduction in the risk of HA-VTE across our 5 academic medical centers. This represents avoidance of significant clinical morbidity (an estimated 81 PEs and 89 DVTs per year) and significant cost. Assuming costs of $\$ 9250$ per DVT and $\$ 13,050$ per PE, ${ }^{17}$ the estimated short-term cost savings are almost $\$ 1.9$ million per year (minus expenditures on VTEP). Further savings might be expected over a longer time horizon because of the avoidance of recurrent VTE, post-thrombotic syndrome, and the costs and complications of longterm anticoagulation.

We believe the highly variable degree of improvement seen across our 5 sites was due to the relatively mature VTEP efforts at the onset of this collaborative improvement effort at UCSD and UCSF. As we noted earlier, the interventional bundle and methods were derived from earlier work at UCSD that had already demonstrated published marked improvement in prophylaxis and a $40 \%$ decrease in HA-VTE. ${ }^{14}$ The narrow range of low HA-VTE rates in 2014 (the mature intervention time period) suggests there may be some HA-VTE rate beyond which further prevention efforts are less productive.

Our study has several limitations. As a longitudinal collaborative improvement effort introducing a bundle of interventions, we cannot ascribe improved outcomes to individual components in the bundle; for example, we did not record the number of measurevention calls or resulting prophylaxis changes. We also did not measure adverse events due to VTEP, believing benefits to be greater than risks, but some adverse events likely did occur and attenuated benefits 
and cost savings. Although we had rigorous measures to assess the prevalence of appropriate prophylaxis, we failed to capture the baseline rate of VTEP, which means we cannot show that improved HA-VTE rates corresponded to improvements in VTEP rates. The bundle of interventions was not implemented uniformly. Some metrics, like adherence to mechanical prophylaxis, were monitored in a decentralized fashion, without collation or collective analysis.

Were improved VTE rates due to decreases in HAVTE detection? We could not detect postdischarge HAVTE that presented to other hospitals, but we have no reason to think the proportion of missed HA-VTE changed over the study. We discouraged the practice of routinely extending duplex ultrasound testing below the knee, and also discouraged surveillance of asymptomatic patients with Doppler ultrasound. This raises the question of ascertainment bias. Did we have fewer HA-VTE in 2014 because our interventions worked, or did we reduce how aggressively we looked for HAVTE? Higher frequencies of ultrasound testing are correlated with higher rates of DVT because of surveillance bias. ${ }^{18}$ Although some reduction in DVT was due to changes in ultrasound practices, several factors suggest the majority of improvement resulted from our interventions. First, only 1 of our 5 sites (UCD) routinely extended ultrasound testing below the knee in the baseline period. Second, we distinguished distal DVT from proximal/unspecified DVT, and the rates of both showed significant improvement. Screening asymptomatic patients with ultrasounds for DVT was limited to a few services in special circumstances (for example, the trauma service at UCSD screened patients at highest risk who could not be prophylaxed with anticoagulation). We did not have the capability to formally track which patients were being diagnosed with screening exams versus for symptoms, but screendetected patients were a small minority. We did not successfully dissuade these few services from stopping this approach, but we did head off some services that were considering this strategy, and think it likely that at best, we kept screening from spreading. Third, PE was reduced by over $20 \%$, in addition to reductions in DVT, even though several of our sites acquired computed tomography scanners more sensitive for small thrombi/incidental PE. Finally, the aggressiveness of ultrasound testing often goes up with aggressive prevention efforts, which would have led to surveillance bias with increased-rather than decreased-rates of HA-VTE.

Our study has a number of strengths. Our effort encompassed a large and inclusive adult inpatient population over a long period of observation, with a relatively large reduction in HA-VTE. These reductions occurred even though the proportion of patients with cancer (our most powerful predictor of VTE risk) was $34.8 \%$ in 2014 versus $33.3 \%$ in 2011. Our metrics captured patients readmitted to the hospital within 30 days of a prior VTE-free admission as well as patients suffering VTE during the hospital stay, with the limitation that we captured only patients readmitted back to our own institutions. Our metrics for VTEP scrutinized prophylaxis rates at different points during hospitalizations, and risk-appropriate prophylaxis was assessed, in contrast to some common regulatory measures that monitor only whether any prophylaxis is in place on the first day of admission or transfer. ${ }^{11}$

Our study should be instructive in terms of focusing improvement efforts. The rate of HA-VTE was much higher in cancer and surgical patients than in medical patients, and we only achieved a nonsignificant $10 \%$ reduction in risk among medical patients (RR: 0.90, 95\% CI: $0.73-1.11$ ). This is consistent with literature demonstrating a more limited benefit of prophylaxis in medical inpatients. ${ }^{19}$ Although we continue to recommend prophylaxis in high-risk medical inpatients, efforts targeting cancer and surgical populations are likely to yield greater results.

Our collaborative used methods that are portable, sustainable, and provide an excellent platform for spread of improvement across a system. The portability of these strategies is underlined by the variable baseline performance and the different stages of electronic health record development at our unique sites. Toolkits that describe the interventions (such as order sets, educational tools, measures, measure-vention) are freely available, and reflect established guidelines. ${ }^{1}$ Our collaborative model is consistent with successful models published in the literature. ${ }^{1,14,20}$ In these models, clinical experts distill the evidence down into key best practices, and design processes that need to occur with the lowest barriers to use. Metrics, expert advice, and toolkits are assembled centrally, while each hospital identifies local barriers to implementation, educates and engages staff, executes implementation, and continually evaluates performance, modifying interventions accordingly. Embedding clinical decision and risk-assessment into VTE prevention modules within commonly used order sets and documentation tools helps to hard-wire the interventions, tightly linking risk assessment to appropriate prophylaxis options. The approach to standardization allows for flexibility for special populations and special needs of unique patients, while minimizing needless variation based on the ordering providers. Program management tools and regular webinars keeps sites on track, coordinate interventions, sustain enthusiasm, and provide a venue for sharing tools and lessons learned. Multiple active interventions are utilized rather than relying on passive educational techniques or order sets alone. Active surveillance (i.e., measure-vention) deserves special attention. Measure-vention has demonstrated utility in inpatient glycemic control and a variety of hospitalassociated infections in addition to VTE prevention, and some systems now uses measure-ventionists as the 
lynchpin for a whole host of successful improvement programs. ${ }^{12,14,21,22}$ We believe high-quality metrics, standardized protocol-driven order sets, and measurevention are the crucial elements for success.

\section{CONCLUSIONS}

Hospital systems can reduce HA-VTE by implementing a bundle of active interventions including standardized VTEP orders with embedded risk assessment and measure-vention. Good measurement of HA-VTE, appropriate VTEP that exceeds minimum regulatory standards, and a robust collaborative infrastructure inform and accelerate improvement. Surgical and cancer populations are at higher risk for HA-VTE and should be a prime focus of improvement efforts.

\section{Disclosures}

Ian H Jenkins: nothing to report. Alpesh $\mathrm{N}$. Amin: nothing to report. Nasim Afsarmanesh: nothing to report. Dr. Auerbach receives honorarium as Editor-in-Chief of the Journal of Hospital Medicine. Dr. Khanna has licensed technology to the hospital-based electronic messaging vendor Voalte and will benefit financially from its dissemination. This does not impact this work. Dr. Maynard acts as a consultant on an expert panel overseeing a multinational trial of extended VTE prophylaxis in high-risk medical patients (Medically Ill Patient Assessment of Rivaroxaban Versus Placebo in Reducing Post-Discharge Venous ThromboEmbolism Risk), a study funded by Johnson \& Johnson. Dr. White has acted as a consultant for Janssen, Boehringer-Ingleheim, Diiachi-Sankyo, and Bristol Meyer Squibb, and provides expert testimony for various malpractice defense lawyers for VTE, and has a grant with the Gordon and Betty Moore Foundation regarding VTE prevention.

\section{References}

1. Maynard G. Preventing Hospital-Associated Venous Thromboembolism: A Guide for Effective Quality Improvement. 2nd ed. Rockville, MD: Agency for Healthcare Research and Quality; October 2015. AHRQ Publication No. 16-001-EF. Available at: http://www.ahrq. gov/professionals/quality-patient-safety/patient-safety-resources/ resources/vtguide/index.html. Accessed June 1, 2016.

2. Heit JA, O'Fallon WM, Petterson TM, et al. Relative impact of risk factors for deep vein thrombosis and pulmonary embolism. Arch Intern Med. 2002;162:1245-1248.

3. Tapson VF, Hyers TM, Waldo AL, et al. Antithrombotic therapy practices in US hospitals in an era of practice guidelines. Arch Intern Med. 2005;165:1458-1464.

4. Kahn SR, Lim W, Dunn AS, et al. Prevention of VTE in nonsurgical patients. Chest. 2012;141(2 suppl):e195S-e226S.

5. Gould MK, Garcia DA, Wren SM, et al. Prevention of VTE in nonorthopedic surgical patients. Chest. 2012;141(2 suppl):e227S-e277S.

6. Falck-Ytter Y, Francis CW, Johanson NA, et al. Prevention of VTE in orthopedic surgery patients. Chest. 2012;141(2 suppl):e278S-e325S.
7. Monreal M, Kakkar A, Caprini J, et al. The outcome after treatment of venous thromboembolism is different in surgical and acutely ill medical patients. Findings from the RIETE registry. I Thromb Haemost. 2004;2:1892-1898.

8. Amin A, Stenkowski S, Lin J, Yang G. Inpatient thromboprophylaxis use in U.S. hospitals: adherence to the Seventh American College of Chest Physician's recommendations for at-risk medical and surgical patients. J Hosp Med. 2009; 4:E15-E21.

9. Centers for Medicare \& Medicaid Services. Fiscal Year 2015 final rules tables. Available at: https://www.cms.gov/Medicare/MedicareFee-for-Service-Payment/AcuteInpatientPPS/FY2015-IPPS-Final-RuleHome-Page-Items/FY2015-Final-Rule-Tables.html. Accessed April 19, 2016.

10. Agency for Healthcare Research and Quality. AHRQ quality indicators ICD-9-CM and ICD-10-CM/PCS specification enhanced version 5.0. patient safety indicator \#12 (PSI \#12)-perioperative pulmonary embolism or deep vein thrombosis rate. Available at: http://www. qualityindicators.ahrq.gov. Accessed April 16, 2016.

11. The Joint Commission. Venous thromboembolism. Available at: http://www.jointcommission.org/venous_thromboembolism. Accessed April 19, 2016.

12. Maynard G, Morris T, Jenkins I, et al. Optimizing prevention of hospital acquired venous thromboembolism: prospective validation of a VTE risk assessment model. J Hosp Med. 2010;5(1):10-18.

13. Harris PA, Taylor R, Thielke R, et al. Research electronic data capture (REDCap) - a metadata-driven methodology and workflow process for providing translational research informatics support. I Biomed Inform. 2009;42:377-381.

14. Maynard GA, Budnitz TL, Nickel WK, et al. Mentored implementation: building leaders and achieving results through a collaborative improvement model. 2011 John M. Eisenberg Patient Safety and Quality Award, National Level. Jt Comm J Qual Patient Saf. 2012; 38(7):301-310.

15. Khanna RR, Kim SB, Jenkins I, et al. Predictive value of the POA indicator for hospital-acquired venous thromboembolism. Med Care. 2013:53(4):e31-e36.

16. Sadeghi B, White RH, Maynard G, et al. Improved coding of postoperative deep vein thrombosis and pulmonary embolism in administrative data (AHRQ patient safety indicator 12) after introduction of new ICD-9-CM diagnosis codes. Med Care. 2015:53(5):e37-e40.

17. Dobesh PP. Economic burden of venous thromboembolism in hospitalized patients. Pharmacotherapy. 2009;29(8):943-953.

18. Bilmoria KY, Chung J, Ju MH, et al. Evaluation of surveillance bias and the validity of the venous thromboembolism quality measure. JAMA. 2013;310(14):1482-1489.

19. Lederle FA, Zylla D, MacDonald R, et al. Venous thromboembolism prophylaxis in hospitalized medical patients and those with stroke: a background review for an American College of Physicians clinical practice guideline. Ann Intern Med. 2011;155(9):602-615.

20. Pronvost PJ, Berenholtz SM, Needham DM. Translating evidence into practice: a model for large scale knowledge translation. BMJ. 2008; 337:a1714

21. Maynard G, Kulasa K, Ramos P, et al. Impact of a hypoglycemia reduction bundle and a systems approach to inpatient glycemic management. Endocr Pract. 2015;21(4):355-367.

22. Jayanthi A. Zero adverse events: how Dignity Health achieved a new standard. Becker's Hospital Review: Infection Control and Clinical Quality website. Available at: http://www.beckershospital review.com/quality/zero-adverse-events-how-dignity-health-achieveda-new-standard.html. Accessed April 19, 2016. 